\title{
PYRAZINAMIDE INDUCED ACUTE GOUTY ARTHRITIS
}

\author{
Pathak U N', Shrestha B $0^{1}$, Shrestha R $\mathbf{R}^{1}$
}

\section{ABSTRACT}

A typical case of pyrazinamide induced acute gouty arthritis in a tuberculous patient with antitubercular therapy including pyrazinamide was reported. It highlights the need of awareness among physicians about the potentially morbid complications of pyrazinamide. The other antitubercular drug like ethambutol may also cause increase in serum uric acid.

Key Words: Gout, Pyrazinamide.

\section{INTRODUCTION}

Gout is an inflammatory arthritis associated with hyperuricemia. The prevalence of gout in Europe and the USA is approximately $0,2 \%$, and it is still increasing mainly in developed countries. ${ }^{1}$ A number of pharmacological agents including pyrazinamide can induce: hyperuricemia and sometimes gout. Although pyrazinamide is routinely and widely used in initial phase of antitubercular therapy in Nepal, pyrazinamide induced acute gouty arthritis is rarely reported. So a typical case of pyrazinamide induced gouty arthritis in a tuberculous patient taking antitubercular therapy is reported to highlight potentially morbid side effects of pyrazinamide.

\section{CASE REPORT}

A 34 years old female from Kathmandu, a case of sputum positive tuberculous patient on Category I of antitubercular therapy for last three weeks, had presented in Medical OPD with complaints of severe pain on first metatarsophalangeal joint at night. There was no history of intake of alcohol, diuretics, excessive exercise or trauma. No such episode was present in the past.

On examination, the patient was thinly built. Her right big toe was swollen, red and hot at the metatarsophalangeal joint with shiny overlying skin. It was excruciatingly tender.

1. Nepal Medical College Teaching Hospital, Attarkhel, Jorpati, Nepal.

Address for correspondence : Prof. Dr. U. N. Pathak, Dept. of Medicine

Nepal Medical College Teaching Hospital, Attarkhel, Jorpati

G.P.O. Box: 13344, Kathmandu, Nepal. 
Initial investigations of blood showed leucocytosis with raised USR. Serum uric acid was $7.2 \mathrm{mg} / \mathrm{dl}$.

The patient was immediately managed with nonsteroidal anti-inflammatory drug - cap indomethacin $25 \mathrm{mg}$ thrice daily for five days but no response was seen. Thereafter tab pyrazinamide was discontinued. After three days, she was completely symptom-free. Two weeks after discontinuation of pyrazinamide repeat serum uric acid came to be $6.0 \mathrm{mg} / \mathrm{dl}$.

\section{DISCUSSION}

Gout is a metabolic disease in which crystals of monosodium urate monohydrate derived from hyperuricemic body fluids give rise to inflammatory arthritis, tenosynovitis, bursitis or cellulitis, tophaceous deposits, urolithiasis and renal disease. ${ }^{2,3}$ The prevalence of gout from study in Europe and the USA is reported to be $0.2 \%$ and it is said to be increasing specially m developed and developing countries. Although the prevalence is influenced by genetic factors, the associations of alcohol consumption, obesity, and hypertension appeal to be partially responsible for the increased prevalence of gout and hyperuricemia in African and Oriental countries. ${ }^{4}$ It may hold true for increasing prevalence of gout in our region.

Gouty arthritis is predominantly a problem of postpubertal males and seldom seen in pre-menopausal females. Hyperuricemia is necessary but not sufficient for the development of gouty arthritis. In many patients, there is often both increased production and decreased excretion of urate. Acute gouty arthritis may be precipitated by several events - dietary excess, trauma, surgery, excessive ethanol ingestion, adrenocorticotrophic hormone (ACTH), glucocorticoid withdrawal, hypouricemic therapy and serious medical illness such as myocardial infarction and stroke. ${ }^{3}$

A number of pharmacological agents can induce hyperuricemia and sometimes gout usually by interfering with the renal tubular excretion of urate but also in some instances by increasing the formation of uric acid. Some of the commonly used drugs which can cause hyperuricemia are low dose salicylates, pyrazinamide, ethambutol, nicotinic acid, cyclosporin, 2-ethylammo-1, 3, 4-thiadiazole fructose and cytotoxic agents. ${ }^{5}$ However, druginduced hyperuricemia and gout are not commonly reported, although these drugs have been widely and commonly used.

In a study carried out at National Minami_kyoto Hospital in Japan by Inove $\mathrm{T}$ et al among 51 pulmonary tuberculous patients on pyrazinamide therapy for 2 months, hyperuricemia was observed in $86 \%$ of cases. Arthralgia was observed in 9 patients, while acute gout was observed in only one patient who had hyperuricemia prior to pyrazinamide treatment. The other 8 patients with arthralgia had symptoms in shoulder and knees, but no gouty pain. ${ }^{6}$ Arthralgia was not related to serum uric acid level and disappeared after stopping pyrazinamide. This study further showed pyrazinamide induced gouty arthritis. Asymptomatic hyperuricemia could be more common.

\section{CONCLUSION}

Acute gouty arthritis can he caused by pyrazinamide therapy and can lead to marked morbidity. Although asymptomatic hyperuricemia is more common with pyrazinamide therapy, possibility of gouty arthritis should be borne in mind by physician and should be explained to the patient. 
REFERENCE

1. D rury $P L$ and S hipley M . C rystal arthritis. I $n K$ umar $P \& C$ lark $M$. editors. C linical $M$ edicine $\left(4^{\text {th }}\right.$ ed. $)$ L ondon: W B S aunders 1998: 482-84.

2. N uki $G, R$ alston $S H, L$ uqmani $R, G$ out. I $n H$ aslettC et al. editors. $D$ avidson's $P$ rinciples and $P$ ractice of $M$ edicine $\left(18^{\text {th }}\right.$ ed.). C hurchill $L$ ivingstone 1999: $831-35$

3. $R$ ginato $A \perp, G$ out and other crystal arthropathies. I $n B$ raunwal $d E$, et al. editors. $H$ arrison's $P$ rinciples of I nternal M edicine ( $15^{\text {th }}$ ed.) M C G raw- $\mathrm{H}$ ill 2001: $1994-95$
4. W ortmann $\mathrm{R} L, G$ out and $\mathrm{H}$ yperuricemia. $\mathrm{C}$ urr $\mathrm{O}$ pin $\mathrm{R}$ heumatol. $2002 \mathrm{M}$ ay; 14(3): 281-6.

5. $S$ cott $J T$. D rug induced gout. $B$ aillieres $C$ lin $\mathrm{R}$ heumatol. $1991 \mathrm{~A} \mathrm{pr;} \mathrm{5(1):} \mathrm{36-39.}$

6. I noveT, I keda N , K urasawa T etal. H yperuricemia and arthralgia during pyrazinamide treatment. $\mathrm{N}$ ihon $\mathrm{K}$ okyuki $\mathrm{G}$ akkai Z asshi. $1999 \mathrm{Feb} ; 37(2)$ : 115-8.

L 2012

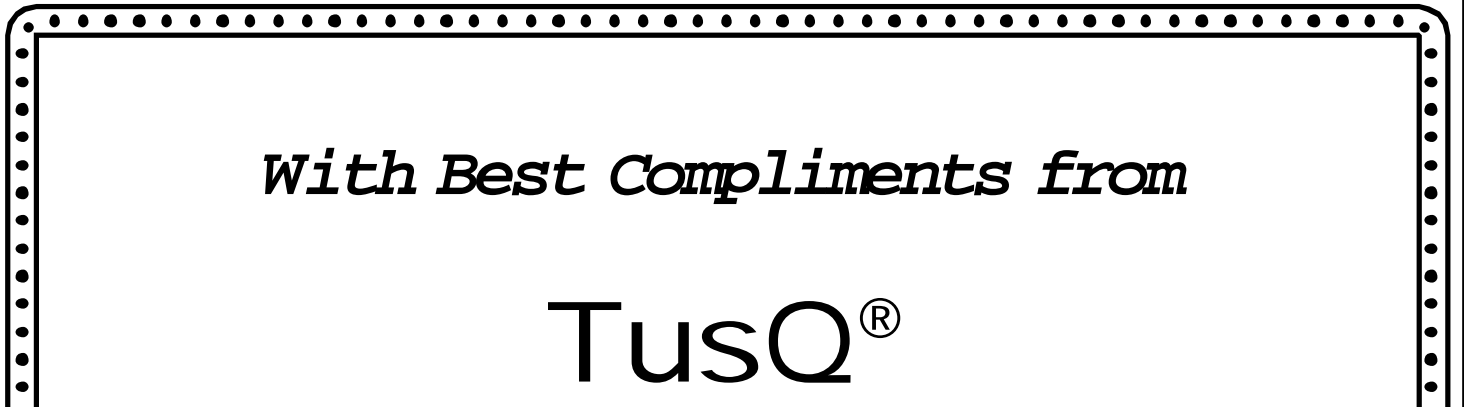

For effective cough control

Unique superior formulations

Ideal viscosity

Longer soothing action

Available As:

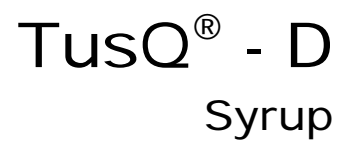

$\operatorname{TusQ}^{\circledR}-\mathrm{X}$ Syrup

TusQ $^{\circledR}$

Tablets

TusQ $^{\circledR}-\mathrm{D}$

TusQ $^{\circledR}$

Lozenges

Oral drops 